\title{
A Fluctuating Line-of-Sight Fading Model with Double-Rayleigh Diffuse Scattering
}

\author{
Jesús López-Fernández, Pablo Ramírez Espinosa, Juan M. Romero-Jerez and F. Javier López-Martínez
}

\begin{abstract}
We introduce the fluctuating double-Rayleigh with line-of-sight (fdRLoS) fading model as a natural generalization of the double-Rayleigh with line-of-sight fading model, on which the constant-amplitude line-of-sight component is now allowed to randomly fluctuate. We discuss the key benefits of the fdRLoS fading model here formulated over the state of the art, and provide an analytical characterization of its chief probability functions. We analyze the effect of the fading parameters that define the model, and discuss their impact on the performance of wireless communication systems.
\end{abstract}

Index Terms-Channel modeling, fading models, Rice, Rician shadowed, second order scattering.

\section{INTRODUCTION}

The ubiquity of wireless systems has been enabled by the combination of multipath propagation, reflection and diffraction that makes communications possible without the existence of a line-of-sight $(\mathrm{LoS})$ between the transmitter and receiver ends. However, in many circumstances the existence of LoS is an inherent characteristic of the propagation conditions in a number of environments, especially as wireless systems move toward higher frequencies [1, 2].

The most popular fading model for LoS propagation is the classical Rician model [3]. It is based on the central limit theorem (CLT), by assuming that a sufficiently large number of multipath components arrive at the receiver end. The direct path is identified with the LoS component and the aggregation of the remaining waves is approximately Gaussian. There are different ways to generalize Rician fading model in order to allow it to model a wider range of propagation conditions. One of such generalizations is the socalled second order scattering fading (SOSF) model introduced in [4] and later formalized in [5], on which an additional term associated to double-scattering was introduced. A special case of this SOSF model is the double-Rayleigh with line-ofsight (dRLoS) fading model, on which the Gaussian diffuse component that arises from the CLT is not considered and the overall scattering corresponds to double-scattering, which in general implies a larger fading severity than its Rayleigh counterpart [6]. Such propagation conditions occur in the context of backscatter communications [7], or in the context of optical wireless communications through the so-called I-K distribution [8]. Further generalizations to incorporate multiple

This work has been funded by the Spanish Government and the European Fund for Regional Development FEDER (project TEC2017-87913-R), and by Junta de Andalucía (project P18-RT-3175).

J. López-Fernández, F. J. López-Martínez and J. M. Romero-Jerez are with Communications and Signal Processing Lab, Instituto Universitario de Investigación en Telecomunicación (TELMA), Universidad de Málaga, CEI Andalucía TECH, ETSI Telecomunicación, Bulevar Louis Pasteur 35, 29010 Málaga (Spain). (contact e-mail: jlf@ic.uma.es).

P. Ramírez-Espinosa is with the Connectivity Section, Department of Electronic Systems, Aalborg University, Aalborg Øst 9220, Denmark. scattering components of increasing order are available in the literature [5, 9], at the expense of a much more sophisticated mathematical formulation.

Another popular generalization of the Rician fading model was introduced by Adbi et al. in [10], by allowing the LoS component to randomly fluctuate. This model, usually referred to as Rician shadowed fading model, has a number of appealing properties: for instance, it does not only generalize Rician fading model by adding an additional parameter that models a more general propagation condition, but in some cases, its mathematical representation is even simpler than the original Rician fading model [11].

The goal of this paper is to introduce a different generalization of Rician fading that includes both LoS fluctuation and second-order scattering at the same time: we refer to this distribution as the fluctuating double-Rayleigh with line-of-sight (fdRLoS) fading model. We will see how the consideration of the random fluctuations in the LoS components allow to alleviate some of the limitations of the baseline dRLoS fading model, such as its peaky behavior that is not representative to real data which causes an overestimation of the peak probability density [12]. We will also analyze how the performance of wireless communication systems operating over fdRLoS fading channels is affected by the different propagation conditions captured by the model.

Notation: $\mathbb{E}\{X\}$ and $|X|$ denote the statistical average and the modulus of the complex random variable (RV) $X$ respectively. The RV $X$ conditioned to $Y$ will be denoted as $X \mid Y$. The symbol $\sim$ reads as statistically distributed as. The symbol $\stackrel{d}{=}$ reads as equal in distribution. A circularly symmetric normal RV $X$ with mean $\mu$ and variance $\Omega$ is denoted as $X \sim \mathcal{N}_{c}(\mu, \Omega)$.

\section{PHYSICAL MODELS}

For the sake of comprehensiveness, and in order to allow for a better understanding of the relationship between the fdRLoS fading model with other state-of-the-art alternatives, we briefly detail in the sequel the physical models for the most relevant amplitude-based LoS fading models in the literature.

\section{A. Physical model for Rician fading}

The physical model for the received signal $S$ under the Rician fading model [3, 13] is given by

$$
S=\omega_{0} e^{j \phi}+\omega_{1} G_{1},
$$

where $\omega_{0} e^{j \phi}$ is the LoS component with average amplitude $\omega_{0}, \phi$ is a RV uniformly distributed in $[0,2 \pi)$, and $G_{1}$ is a zero-mean normal complex Gaussian RV, i.e. $G_{1} \sim \mathcal{N}_{c}(0,1)$. The weighting factor $\omega_{1}$ is real and non-negative, and scales the variance of $G_{1}$. Besides its average power $\Omega=\mathbb{E}\left\{S^{2}\right\}=$ 
$\omega_{0}^{2}+\omega_{1}^{2}$, the Rician model is completely defined by the socalled Rician $K$ parameter, defined as

$$
K=\frac{\omega_{0}^{2}}{\omega_{1}^{2}} \in[0, \infty),
$$

which accounts for the ratio between the powers associated to the LoS and non-LoS components.

\section{B. Physical model for Rician shadowed fading}

The physical model for the received signal $S$ under the Rician shadowed fading model [10] is given by

$$
S=\omega_{0} \sqrt{\xi} e^{j \phi}+\omega_{1} G_{1},
$$

where the parameters in (3) are equivalent to those in (1), and $\xi$ is a Gamma distributed RV with unit power and real positive shape parameter $m$, with probability density function (PDF):

$$
f_{\xi}(u)=\frac{m^{m} u^{m-1}}{\Gamma(m)} e^{-m u},
$$

where $\Gamma(\cdot)$ is the gamma function. This variable $\xi$ captures the LoS fluctuations, ranging from very severe for low $m$ to milder ones as $m$ grows. In the limit case of $m \rightarrow \infty, \xi$ degenerates to a deterministic unitary value and the LoS fluctuation vanishes, then specializing to the conventional Rician fading model.

The Rician shadowed model is characterized by two parameters: $K$, as in (2), and $m \in(0, \infty)$.

\section{Physical model for dRLoS fading}

The physical model for the received signal $S$ under dRLoS fading is expressed as in [5]:

$$
S=\omega_{0} e^{j \phi}+\omega_{2} G_{2} G_{3},
$$

where $\omega_{0}$ and $\phi$ inherit the same definitions as in the Rician model, $G_{2}$ and $G_{3}$ are independent and identically distributed (i.i.d.) zero-mean normal complex Gaussian RVs, i.e. $G_{i} \sim$ $\mathcal{N}_{c}(0,1)$ for $i=2,3$. The component $\omega_{2} G_{2} G_{3}$ leads to a double-Rayleigh (dR) fading component, and hence, it differs from classical Rician fading for which the diffuse component lead to a single-Rayleigh RV.

The parameters $\omega_{0}$ and $\omega_{2}$ are restricted to being real and non-negative. As in the Rician fading model, the parameter $K$ suffices to completely define the distribution, i.e.,

$$
K=\frac{\omega_{0}^{2}}{\omega_{2}^{2}} \in[0, \infty),
$$

The model in (5) includes the $\mathrm{dR}$ fading model when $K=0$.

\section{Physical model for fluctuating dRLoS fading}

The physical model for the received signal $S$ under the dRLoS fading model with LoS fluctuations is a natural generalization of the dRLoS model introduced by Salo et al. [5], as

$$
S=\omega_{0} \sqrt{\xi} e^{j \phi}+\omega_{2} G_{2} G_{3},
$$

where $\omega_{0} e^{j \phi}$ is the LoS component with constant amplitude $\omega_{0}, \phi$ is a RV uniformly distributed in $[0,2 \pi), G_{2}$ and $G_{3}$ are i.i.d. zero-mean normal complex Gaussian RVs, i.e. $G_{i}$ is distributed as $\mathcal{N}_{c}(0,1)$ for $i=2,3$, and $\xi$ is a Gamma distributed $\mathrm{RV}$ with unit power and real positive shape parameter $m$. As in the Rician shadowed fading model, the fdRLoS fading model is completely defined by two parameters $K$ as in (6) and $m$.

\section{Statistical Characterization}

In this section, we will derive analytical expressions for the PDF and the cumulative distribution function (CDF) of the instantaneous signal-to-noise ratio (SNR) $\gamma$ under fdRLoS fading. Assuming a normalized channel with $\mathbb{E}\left\{|S|^{2}\right\}=1$, we have that $\gamma=\bar{\gamma}|S|^{2}$, where $\bar{\gamma}$ is the average SNR. We will base our derivations on the key findings detailed in [6], that will allow us to connect the fdRLoS fading distribution with an underlying Rician shadowed distribution.

Let us begin by considering (7), so that we have

$$
\gamma=\bar{\gamma}\left|\omega_{0} \sqrt{\xi} e^{j \phi}+\omega_{2} G_{2} G_{3}\right|^{2} .
$$

Now, we express the complex Gaussian RV $G_{3}$ as $G_{3}=$ $\left|G_{3}\right| e^{j \Psi}$, where $\Psi$ is uniformly distributed in $[0,2 \pi)$. Because $G_{2}$ is a circularly-symmetric RV, the following equivalence in distribution holds for $\gamma$

$$
\left.\gamma \stackrel{d}{=} \bar{\gamma}\left|\omega_{0} \sqrt{\xi} e^{j \phi}+\omega_{2} G_{2}\right| G_{3}\right|^{2}
$$

Conditioning on $x=\left|G_{3}\right|^{2}$, define the conditioned RV $\gamma_{x}$ as

$$
\gamma_{x} \triangleq \bar{\gamma}|\underbrace{\omega_{0} \sqrt{\xi} e^{j \phi}+\omega_{2} \sqrt{x} G_{2}}_{S_{x}}|^{2} .
$$

We see that $S_{x}$ is a Rician shadowed RV as in (3), with $\omega_{1}=$ $\omega_{2} \sqrt{x}$. Hence, we have that $\gamma_{x}$ is distributed according to a squared Rician shadowed RV with parameters $m$,

$$
\begin{aligned}
\bar{\gamma}_{x} & =\mathbb{E}\left\{\gamma_{x}\right\}=\bar{\gamma}\left(\omega_{0}^{2}+x \omega_{2}^{2}\right)=\bar{\gamma} \frac{K+x}{K+1}, \\
K_{x} & =\frac{\omega_{0}^{2}}{x \omega_{2}^{2}}=\frac{K}{x},
\end{aligned}
$$

where $K=\omega_{0}^{2} / \omega_{2}^{2}$ is the Rician factor in the absence of the RV $x$, i.e., as in 6. Hence, the PDF of $\gamma_{x}$ is that of the SNR of a Rician shadowed RV, i.e. [10, 14],

$f_{\gamma_{x}}(\gamma ; x)=\frac{m^{m}\left(1+K_{x}\right)}{\left(m+K_{x}\right)^{m} \bar{\gamma}_{x}} e^{-\frac{1+K_{x}}{\bar{\gamma}_{x}} \gamma}{ }_{1} F_{1}\left(m ; 1 ; \frac{K_{x}\left(1+K_{x}\right)}{K_{x}+m} \frac{\gamma}{\bar{\gamma}_{x}}\right)$,

where ${ }_{1} F_{1}(\cdot ; \cdot ; \cdot)$ denotes the Kummer confluent hypergeometric function [15, eq. (16.2)]. Noting that $\left|G_{2}\right|^{2}$ is exponentially distributed with unitary mean, we can compute the distribution of $\gamma$ by averaging over all possible values of $x$ as:

$$
f_{\gamma}(\gamma)=\int_{0}^{\infty} f_{\gamma_{x}}(\gamma ; x) e^{-x} d x
$$

Plugging 13 into 14, an integral expression for the PDF of the fdRLoS model is derived. In order to obtain a simpler expression for $f_{\gamma}(\gamma)$, we consider that the parameter $m \in \mathbb{Z}^{+}$. We note that such restriction does not cause a major impact unless the LoS fluctuation is very severe; as we will later see, the practical benefits of the fdRLoS fading model will become apparent precisely for mild and medium fluctuations of the LoS component. Under this premise, the PDF of the Rician shadowed fading model simplifies as [14, eq. (5)]

$$
f_{\gamma_{x}}(\gamma ; x)=\sum_{j=0}^{m-1} \frac{C_{j}(x)}{(m-j-1) !} \frac{\gamma^{m-j-1}}{\Omega(x)^{m-j}} e^{-\frac{\gamma}{\Omega(x)}},
$$

with $C_{j}(x)=\left(\begin{array}{c}m-1 \\ j\end{array}\right)\left(\frac{m x}{m x+K}\right)^{j}\left(\frac{K}{K+m x}\right)^{m-1-j}$ and $\Omega(x)=$ $\bar{\gamma} \frac{K+m x}{m(K+1)}$. Substituting 15 in 14 , using the change of 
variables $t=\frac{1}{m}(K+m x)$ and taking into account that $(t-K / m)^{j}=\sum_{r=0}^{j}\left(\begin{array}{c}j \\ r\end{array}\right) t^{j}(-K / m)^{j-r}$, the following expression for the fdRLoS PDF is derived:

$$
\begin{aligned}
& f_{\gamma}(\gamma)=\sum_{j=0}^{m-1}\left(\begin{array}{c}
m-1 \\
j
\end{array}\right) \frac{(K / m)^{m-j-1}(K+1)^{m-j} e^{K / m}}{\bar{\gamma}^{m-j}(m-j-1) !} \gamma^{m-j-1} \times \\
& \sum_{r=0}^{j}\left(\begin{array}{l}
j \\
r
\end{array}\right)\left(\frac{-K}{m}\right)^{j-r} \Gamma\left(r+j-2 m+2, \frac{K}{m}, \frac{\gamma}{\bar{\gamma}}(K+1)\right),
\end{aligned}
$$

where $\Gamma(a, z, b)=\int_{z}^{\infty} t^{a-1} e^{-t} e^{\frac{-b}{t}} d t$ is a generalization of the incomplete gamma function defined in [16].

The CDF of the fdRLoS fading model can also be obtained by averaging the CDF of $\gamma_{x}$, i.e., the Rician shadowed CDF over the exponential distribution:

$$
F_{\gamma}(\gamma)=\int_{0}^{\infty} F_{\gamma_{x}}(\gamma ; x) e^{-x} d x
$$

For the case of integer $m$, a closed-form expression for the Rician shadowed CDF is presented in [14, eq. (10)], i.e.

$$
F_{\gamma_{x}}(\gamma ; x)=1-\sum_{j=0}^{m-1} C_{j}(x) e^{-x / \Omega(x)} \sum_{r=0}^{m-j-1} \frac{1}{r !}\left(\frac{x}{\Omega(x)}\right)^{r},
$$

Plugging (18) into (17), the following expression for the fdRLoS CDF is obtained:

$$
\begin{aligned}
& F_{\gamma}(\gamma)=1-\sum_{j=0}^{m-1}\left(\begin{array}{c}
m-1 \\
j
\end{array}\right)\left(\frac{K}{m}\right)^{m-j-1} e^{K / m} \times \\
& \sum_{r=0}^{m-j-1} \frac{1}{r !}\left(\frac{\gamma}{\bar{\gamma}}\right)^{r}(K+1)^{r} \times \\
& \sum_{s=0}^{m-j-1}\left(\begin{array}{l}
j \\
s
\end{array}\right)\left(\frac{-K}{m}\right)^{j-s} \Gamma\left(s-m-r+2, \frac{K}{m}, \frac{\gamma}{\gamma}(K+1)\right) .
\end{aligned}
$$

In Fig. 1, we represent the evolution of the PDF of the fdRLoS fading model using (16) as the LoS fluctuation severity $m$ changes, for $K=5$ and $\bar{\gamma}_{\mathrm{dB}}=3 \mathrm{~dB}$ (i.e., $\bar{\gamma}=2$ ). Monte Carlo (MC) simulations are included in all instances to double-check the corresponding theoretical expressions. We see that as $m$ is increased, i.e., the fading severity of the LoS component is decreased, the SNR values are less disperse and lower SNR values are less likely. We see that for large $m$, the PDF of the fdRLoS fading model tends to behave as the PDF of the dRLoS fading model (in black solid line); however, note that the peaky behavior exhibited by the dRLoS fading model does not appear in the fdRLoS case, which has a smoother shape.

\section{Application: Outage Probability}

The outage probability (OP) is defined as the probability that the instantaneous SNR takes a value below a given threshold $\gamma_{\text {th }}$, which is directly computed from the CDF in (19) as OP = $F_{\gamma}\left(\gamma_{\text {th }}\right)$. In the high-SNR regime, an asymptotic expression for the OP can be obtained as follows: since the asymptotic OP under Rician shadowed fading is given by [14]

$$
\mathrm{OP}_{\mathrm{RS}}\left(\gamma_{\mathrm{th}} ; \bar{\gamma}, K, m\right)=t \frac{\gamma_{\mathrm{th}}}{\bar{\gamma}}(1+K)\left(\frac{m}{K+m}\right)^{m},
$$

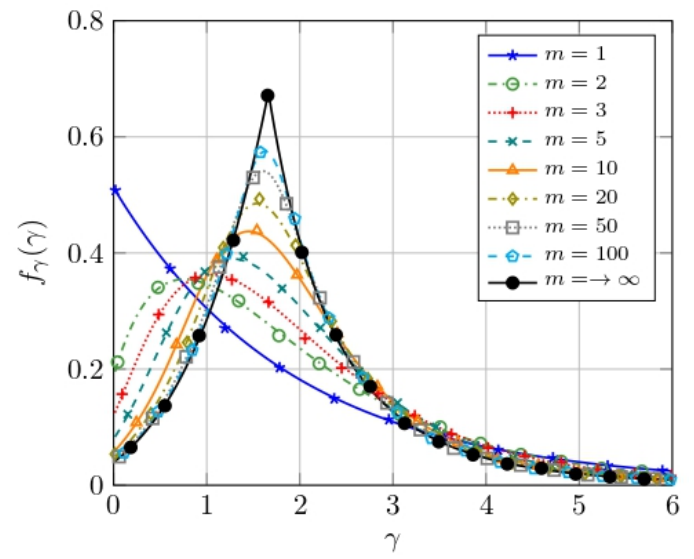

Fig. 1. PDF of the fdRLoS fading model for different values of $m$ as a function of $\gamma$ (in linear form). Parameter values are $K=5$ and $\bar{\gamma}_{\mathrm{dB}}=3 \mathrm{~dB}$. Theoretical values (16) are represented with lines. Markers correspond to MC simulations. The case $m \rightarrow \infty$ is the dRLoS PDF in [6. eq. (15)].

then using the same rationale as in Section III we have that

$$
\begin{aligned}
\mathrm{OP}\left(\gamma_{\mathrm{th}} ; \bar{\gamma}, K, m\right) & =\int_{0}^{\infty} \mathrm{OP}_{\mathrm{RS}}\left(\gamma_{\mathrm{th}} ; \bar{\gamma}(x), K(x), m\right) e^{-x} d x, \\
& =\frac{\gamma_{\text {th }}}{\bar{\gamma}} \underbrace{(1+K) \Gamma(m) \mathrm{U}(m, 1, K / m)}_{a}, \quad(21)
\end{aligned}
$$

where $\bar{\gamma}(x)=\bar{\gamma} \frac{K+k}{K+1}$ and $K(x)=K / x$, and $\mathrm{U}(\cdot, \cdot, \cdot)$ is Tricomi's confluent hypergeometric function [15, (13.1)].

We see that the OP in 21) decays with a diversity order of 1 , while the parameter $a$ acts as power offset or coding gain as defined in [17]. Interestingly, such asymptotic expression is not valid for the specific case of $K=0$ as $\lim _{K \rightarrow 0} \mathrm{U}(m, 1, K / m) \rightarrow \infty$. This corresponds to the $\mathrm{dR}$ or Rayleigh product channel, and for which the assumptions taken in [17] cease to hold, i.e., the OP for the pure $\mathrm{dR}$ case does not behave as $\mathrm{OP}_{\bar{\gamma} \rightarrow \infty}=\frac{a}{t+1}\left(\frac{\gamma_{\text {th }}}{\bar{\gamma}}\right)^{t}$ for any $t>0$. In Fig. 2, OP is depicted for $K=1, \gamma_{\mathrm{th}}=3 \mathrm{~dB}$ and for different values of $m$. As expected, the system performance improves as the LoS fluctuation is reduced, i.e. the OP decreases for higher values of $m$. Observe that for moderate values of $m$ the OP behavior is practically indistinguishable from that of the deterministic LoS (see the zoomed box in the figure). This reveals that the fdRLoS model quickly converges as $m$ grows to the baseline dRLoS starting model, a fact that is not so evident from the PDF curves in Fig 1 .

Next, a comparison between the fdRLoS (fluctuating LoS and double-Rayleigh) and the Rician shadowed (fluctuating LoS and Rayleigh) models is addressed. In the absence of LoS component, it is known that a double-Rayleigh diffuse component leads to a worsening of the system performance as compared to a single-Rayleigh one. Surprisingly, this behavior may change in the presence of a LoS component: in Fig. 3 the OP as a function of $\bar{\gamma}$ is evaluated for the fdRLoS (dashed lines) and Rician shadowed (solid lines) fading models with parameters $K=6, \gamma_{\text {th }}=3 \mathrm{~dB}$ and different values of $m$. See that for $m=\{3,5,10\}$ there is a range of $\bar{\gamma}$ for which the OP of the fdRLoS model falls below that of the Rician shadowed. This counterintuitive behavior is better reflected in 


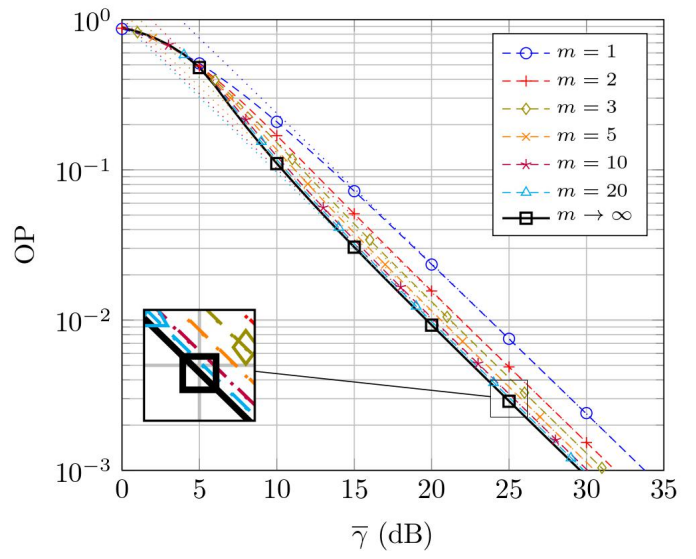

Fig. 2. OP plot. Parameter values are $K=1$ and $\gamma_{\mathrm{th}}=3 \mathrm{~dB}$. Dashed lines correspond to fdRLoS fading. The case with $m \rightarrow \infty$ corresponds to dRLoS fading [6] eq. (21)]. Dotted lines correspond to the asymptotic OP values in 21). Markers correspond to MC simulations.

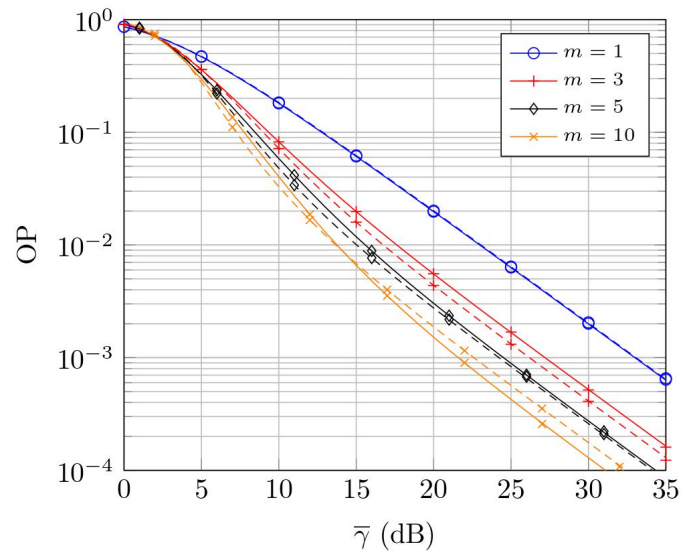

Fig. 3. OP comparison between fdRLoS (dashed lines) and Rician shadowed (solid lines) fading models. OP vs $\bar{\gamma}(\mathrm{dB})$, for different values of $m$. Parameter values are $K=6$ and $\gamma_{\mathrm{th}}=3 \mathrm{~dB}$. Markers correspond to $\mathrm{MC}$ simulations.

Fig 4 where the OP has been plotted as a function of $K$ with fixed parameters $\gamma_{\mathrm{th}}=3 \mathrm{~dB}$ and $\bar{\gamma}=25 \mathrm{~dB}$. First, notice that for $K=0$ (no LoS) the Rician shadowed model effectively yields a lower OP than the fdRLoS model in all instances. As $K$ grows this relation is reverted in a range of $K$ whose size is dependent on $m$. In particular, see that this range is wider for moderate values of $m$, i.e. it is null for $m=1$, grows for $m=3$ and decreases for $m=5$ and $m=10$.

\section{CONClusion}

We introduced and characterized a new family of fading models consisting on a fluctuating LoS component with $\mathrm{dR}$ diffuse component. This model generalizes a class of fading models that arise fom the SOSF model, and also exhibits a smoother behavior than its deterministic LoS counterpart, thus avoiding one of the reported limitations of the dRLoS model. We confirmed that in the presence of a moderate LoS component, the performance of wireless communication systems operating under dR-diffusely scattered fading can be better than for the single-Rayleigh case. This does not happen when the LoS magnitudes are either small or large.

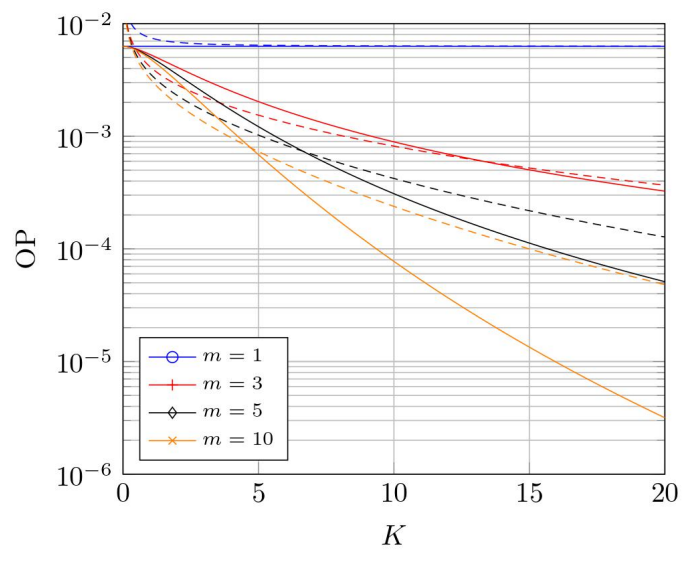

Fig. 4. OP comparison between fdRLoS (dashed lines) and Rician shadowed (solid lines) fading models. OP vs $K$ for different values of $m$. Parameter values are $\bar{\gamma}=25 \mathrm{~dB}$ and $\gamma_{\mathrm{th}}=3 \mathrm{~dB}$.

\section{REFERENCES}

[1] E. Zöchmann, S. Caban, C. F. Mecklenbräuker, S. Pratschner, M. Lerch, S. Schwarz, and M. Rupp, "Better than Rician: modelling millimetre wave channels as two-wave with diffuse power," EURASIP J WIREL COMM, vol. 2019, no. 1, p. 21, 2019.

[2] J. Reig, V. M. Rodrigo Peñarrocha, L. Rubio, M. T. Martínez-Inglés, and J. M. Molina-García-Pardo, "The folded normal distribution: A new model for the small-scale fading in line-of-sight (los) condition," IEEE Access, vol. 7, pp. 77328-77339, 2019.

[3] S. O. Rice, "Mathematical analysis of random noise," Bell Labs Tech. $J$, vol. 24, no. 1, pp. 46-156, 1945.

[4] J. B. Andersen, "Statistical distributions in mobile communications using multiple scattering," in Proc. 27th URSI General Assembly, pp. 1-4, 2002.

[5] J. Salo, H. M. El-Sallabi, and P. Vainikainen, "Statistical Analysis of the Multiple Scattering Radio Channel," IEEE Trans. Antennas Propag., vol. 54, pp. 3114-3124, Nov 2006.

[6] J. Lopez-Fernandez and F. J. Lopez-Martinez, "Statistical Characterization of Second-Order Scattering Fading Channels," IEEE Trans. Veh. Technol., vol. 67, pp. 11345-11353, Dec 2018.

[7] J. K. Devineni and H. S. Dhillon, "Ambient Backscatter Systems: Exact Average Bit Error Rate Under Fading Channels," IEEE Trans. Green Commun. Netw., vol. 3, no. 1, pp. 11-25, 2019.

[8] L. C. Andrews and R. L. Phillips, "I-K distribution as a universal propagation model of laser beams in atmospheric turbulence," J. Opt. Soc. Am. A, vol. 2, pp. 160-163, Feb 1985.

[9] V. Nikolaidis, N. Moraitis, P. S. Bithas, and A. G. Kanatas, "Multiple Scattering Modeling for Dual-Polarized MIMO Land Mobile Satellite Channels," IEEE Trans. Antennas Propag., vol. 66, pp. 5657-5661, Oct 2018.

[10] A. Abdi, W. C. Lau, M. . Alouini, and M. Kaveh, "A new simple model for land mobile satellite channels: first- and second-order statistics," IEEE Trans. Wireless Commun., vol. 2, no. 3, pp. 519-528, 2003.

[11] J. F. Paris, "Closed-form expressions for Rician shadowed cumulative distribution function," Electron. Lett., vol. 46, pp. 952 -953, June 2010.

[12] J. H. Churnside and R. G. Frehlich, "Experimental evaluation of $\log$ normally modulated Rician and IK models of optical scintillation in the atmosphere," J. Opt. Soc. Am. A, vol. 6, pp. 1760-1766, Nov 1989.

[13] M. K. Simon and M.-S. Alouini, Digital communication over fading channels, vol. 95. John Wiley \& Sons, 2005.

[14] J. F. Paris, "Statistical Characterization of $\kappa-\mu$ Shadowed Fading," IEEE Trans. Veh. Technol, vol. 63, pp. 518-526, Feb 2014.

[15] "NIST Digital Library of Mathematical Functions." http://dlmf.nist.gov/, Release 1.0.21 of 2018-12-15. F. W. J. Olver, A. B. Olde Daalhuis, D. W. Lozier, B. I. Schneider, R. F. Boisvert, C. W. Clark, B. R. Miller and B. V. Saunders, eds.

[16] M. Chaudhry and S. Zubair, "Generalized incomplete gamma functions with applications," J Comput Appl Math, vol. 55, no. 1, pp. 99 - 123, 1994.

[17] Zhengdao Wang and G. B. Giannakis, "A simple and general parameterization quantifying performance in fading channels," IEEE Trans. Commun., vol. 51, no. 8, pp. 1389-1398, 2003. 